\title{
Bacterial Identification in Symptomatic and Asymptomatic Endodontic Infections by Culture Method
}

\author{
Bharati D Deo ${ }^{1}$, Shashikala $\mathrm{K}^{2}$, Kishore G Bhat ${ }^{3}$ \\ 1. Dept. of Conservative Dentistry Govt. Dental College and Research Institute, Bangalore India \\ 2. Dept of Conservative Dentistry, DAPMRV Dental College, Bangalore, India \\ 3. Maratha Mandal's NGH Dental College and Research Institute, Belgaum, India
}

\begin{abstract}
The aim of this study was to investigate the microorganisms from of necrotic root canals and to study the associations between symptoms and certain bacterial species, using culture methods. Nineteen symptomatic and twenty-one asymptomatic cases, between 18 to 75 years, having primary endodontic infections, and adhering to the inclusion and exclusion criteria were selected for this study. The targeted microorganisms were Prevotella, Porphyromonas, A.actinimycetmcomitans. E.faecalis.. The organisms that were detected were, Porphyromonas gingivalis, Aggregatibactor actinomycetemcomitans, Prevotella and additional important bacteria like Streptococci, Fusobacterium nucleatum, Klebsiella Capnocytophaga were found by culture analysis, but microorganisms such as and E. faecalis could not be identified. No association was seen between symptoms and bacterial species detected.
\end{abstract}

Keywords: culture, endodontic infection, microbial identification.

\section{Introduction}

Microorganisms and their products are etiological factors for pulp and periapical disease ${ }^{1}$. The goal of endodontic treatment is the eradication of these microbes. Current treatment protocols like isolation, canal preparation, antibacterial irrigants and intracanal medicaments, are directed towards bacterial elimination. The rationale for the development of treatment protocols are all based on data involving microbiological root canal sampling (MRS) as there is an intimate involvement of bacteria and clinical endodontics. Microbiological root canal sampling is the foundation of clinical endodontics. ${ }^{2}$ The finding from microbiologic studies conducted at different parts of the globe reveal that a given species that is very prevalent in root canals of patients from some geographic regions is not necessarily found in similar figures or even detected in samples from other locations. ${ }^{3}$ Studies have suggested that genetic and environmental factors can influence the composition of oral microbiota particularly with regard to the periodontal pathogens. ${ }^{4,5}$ It is well known that most periodontal pathogens are also endodontic pathogens and as a consequence variations in their prevalence have a potential to exert a significant influence in the composition of the microbiota of infected root canals. Similar variations were seen in microflora of endodontic infections. ${ }^{6}$

Endodontic infection can be symptomatic or asymptomatic and the factors responsible for the development of symptoms are not completely understood. Early studies attempted to correlate some bacterial species with symptoms, but it has been demonstrated that the same species can also be found in asymptomatic cases. It seems that the appearance of symptoms might be a result of a complex mechanism, depending on diverse pathogen related and host-related factors.

Despite the availability of various molecular methods for the evaluation of microbes, culture analyses are not redundant. The advantage of cultivation approaches are related to their broad-range nature, which makes it possible to identify a great variety of microbial species in a sample, including those that are not being sought after. Cultivation makes it possible to study the physiological properties and pathogenicity of the microbes. There has been a significant focus recently on developing methods for the in-vitro cultivation of those species that were till recently refractory to culture. Use of dilute nutrient media, increasing the incubation time, modification of the culture media with addition of specific substrates, co-cultivation with helper strains, colony hybridization method, used in isolation or in combination have helped in the culturing of hitherto uncultivable microbes. $^{7}$ An effort has been made in this study to identify the microbes in symptomatic and asymptomatic primary endodontic infections in patients, by the culture method.

\subsection{Patient Selection.}

\section{Materials and Methods}

After approval from the Institutional Ethical Board, of the RV Dental College, Bangalore, Karnataka, India, and obtaining a written informed consent from the selected cases before sampling, forty adult patients participated in the study. The cases were between 18 and 75 years of age. Twenty male and twenty female patients, were selected for the study. Teeth included in this study were from patients presenting with irreversible 
pulpitis and requiring endodontic treatment. Patients with systemic diseases like diabetes mellitus, cardiovascular diseases, immune- compromised cases and patients with periodontal pockets and advanced bone loss as well as patients on prior antibiotic therapy for 2 months were not included. Nineteen patients had symptoms of spontaneous or provoked pain exacerbated by mastication or mild tenderness to percussion and twenty-one patients were asymptomatic

\section{2,2. Microbial Sampling}

Strict asepsis was followed during sample collection. Briefly after the administration of local anesthesia, the tooth was isolated with rubber dam. The tooth and the dam along with the clamp were scrubbed with $3 \%$ hydrogen peroxide and with $2.5 \%$ sodium hypochlorite for one minute followed by inactivation with $5 \%$ sodium thiosulphate. Sterility check was carried out to prove the efficacy of the aseptic technique. Caries and/or existing restorations, if present were removed and then the cavity was wiped with a sterile cotton pellet slightly wet with $2.5 \%$ sodium hypochlorite and then inactivated with $5 \%$ sodium thiosulphate. The pulp chamber was then accessed with a sterile bur, using sterile saline solution for irrigation. A sterile no. $15 \mathrm{k}$ file was introduced in the canal upto $3 \mathrm{mms}$. short of the apex as assessed by the preoperative radiograph and the canals briefly instrumented. If purulent or serous fluid was present in the canal, this was directly sampled with the paper points, (placed in the canal for 30 seconds). Otherwise sterile saline was deposited in the canal and a $\mathrm{K}$ file depending on the size of the canal was introduced to the working length and canals briefly instrumented and then the paper points were used to take the sample. The samples were collected in tubes containing reduced transport fluid (RTF) and were transferred to the laboratory.

\section{2,3. Microbial Selection}

Selection of target microorganisms from the root canals was based on the following criteria: organisms that are frequently found in endodontic infections (Prevotella. and Porphyromonas), organisms that have been detected from root canals from patients who have failed endodontic treatment (Enterococcus spp.) but have also been detected in some cases of primary endodontic infections and organisms that were most commonly seen in periodontal infections but have also been seen in root canals (Aggregatibactor actinomycetemcomitans).

\subsection{Microbial Isolation}

Samples were processed anerobically by using modified gas pack anaerobic jar. Media for microorganism culture were as follows:

Enriched culture medium, Blood agar medium with hemin and vitamin-k for E.faecalis and for most micro-organisms.and Selective culture media:-Dentaid media for Aggregatibactor actinomycetecomitans, Kanamycin blood agar medium for Porphyromonas gingivalis and other Porphyromonas, KanamycinVancomycin blood agar for Prevotella. The plates were incubated in 48 to 72 hours in anaerobic jar.at $37^{\circ} \mathrm{C}$. Later the organisms were identified by colony morphology, Gram stain, pigmentation, growth on selective media and biochemical reactions. All the media used, and the anerobic jar were from procured from the company, Himedia. The colony forming units were counted for the samples and are presented in TABLE-1, Irreversible pulpitis with symptoms and TABLE-2, Irreversible pulpitis without symptoms. TABLE-3, percentage of microbes detected in the samples.

\section{Statistical Analysis}

The detection of target organisms was recorded as the percentage of cases examined in symptomatic and asymptomatic groups. Further we investigated if there was any influence of age, by comparing the organisms in cases below and above 30 years of age, and if there was any influence of gender on the number of organisms detected. The collected data was analyzed by application of Mann-Whitney test at the level of significance: $\alpha=0.05$ ( $\mathrm{P}<0.05)$. The results showed that there is no significant difference in the mean number of organisms detected between the two groups. Age and gender did not play any role in the mean number of organisms detected, between the two groups.

\section{Results}

Of the forty samples collected, thirty-three samples tested positive for the presence of microorganisms and seven samples showed no growth as revealed by anaerobic culture method. Fifteen cases from the symptomatic and eighteen cases from the asymptomatic group showed growth of organisms. The appropriate media were used for growth of organisms and they were identified by colony morphology, Gram stain, pigmentation, growth on selective media and key biochemical reactions. The samples were divided into two groups, Group-I, primary endodontic infection with symptoms and Group-II primary endodontic infections without symptoms. The organisms detected were Streptococci, Porphyromonas gingivalis, Prevotella, Aggregatibactor actinomycetemcomitans, Fusobacterium nucleatum, Klebsiella, Capnocytophaga and 
contamination with Pseudomonas. The sample, the organisms detected and the number of colony forming units were assessed for both the groups, refer Table -1 and Table -2 respectively. The percentage of cases in which these organisms were detected in each group is also tabulated, refer Table-3 and is depicted in the graph (Fig.I). In Group I, from symptomatic irreversible pulpitis group, the prevalence of various organisms is as followas:Streptococci-17/19 (89.4.\%), Porphyromonas gingivalis-7/19 (36.8\%), Prevotalla-7/19 (36.8\%), Fusobacterium nucleatum-8/19 (42.1\%), Aggregatibactor actinomycetemcomitans -3/9 (15.8\%) In Group II, from asymptomatic irreversible pulpitis cases the prevalence of various organisms is as follows-Streptococcii15/21(71.4.0\%), Porphyromonas gingivalis-10/21(47.6\%), Prevotella-2/21(9.5\%), Fusobacterium nucleatum6/21(28.5\%), Aggregatibactor actinomycetemcomitans-9/21(42.8\%) , Klebsiella-1/21 (4.7\%), Capnocytophaga-1/21(4.7\%) and contamination with Pseudomonas in one case. E.faecalis was not detected in any of the forty samples.

\section{Discussion}

Endodontic infection is the major etiological factor in the apical periodontits. Although chemical and physical factors can induce periradicular inflammation, micro-organisms are essential to the progression and perpetuation of apical periodontitis. Endodontic infections are polymicrobial and obligate anaerobes dominate the microbiota of primary intraradicular infections. The endodontic microbiota presents a high inter-individual variation, that is, it can significantly vary in species diversity and abundance from individual to individual and also in individuals from different geographical locations. Understanding the endodontic microbiota is an important requirement for researchers as well as the clinicians so that effective treatment strategies can be followed. Culturing methods are considered the gold standard, used in research. Due to development of new technologies, identification of pathogens also involves microscopy, immunological assays and molecular methods. However culturing as a method, measures viable bacterial cells as colony-forming units while molecular methods measure nucleotide sequences and viable microorganisms are not required. Cultivation discloses a wider spectrum of microorganisms in root canal system. By using this method it is easier to detect contamination of the sample during laboratory manipulation. Viability of microorganism cells could be detected and these microorganisms could be used further with other tests and in research studies.

In this study, samples were collected from the root canals of forty healthy adults (both male and female) cases, with primary endodontic infections nineteen cases were symptomatic and twenty-one cases were asymptomatic. Samples were processed anaerobically by culturing in selective media and identifying the organisms and counting the same. Recently molecular techniques have been used in several studies for bacterial identification, however culture is the gold standard for bacterial detection and identification. Hence the culture technique was followed in this study.

The data collected from the study are shown in TABLE-1 AND TABLE-2. Of the forty samples tested, thirty-three (seventeen from symptomatic and sixteen from asymptomatic group) tested positive for microbial culture. Streptococci were detected in $89.4 \%$ and $71.4 \%$ of samples from symptomatic and asymptomatic cases respectively and in large numbers, (CFU/ml). This is in accordance with the previous studies by Sundqvist ${ }^{1}$ and Siqueira et al. ${ }^{3 .}$ In a study by Skucaite et al. ${ }^{8}$ wherein culture was used for detection of the microbes in primary endodontic infections with symptomatic apical periodontitis, Streptococci were the predominant type of microorganisms detected.

In our study, the targeted organisms, Porphyromonas gingivalis was detected in $36.8 \%$ of symptomatic and in $47.6 . \%$ of asymptomatic cases. As reported in a study by Gomes et $\mathrm{al}^{9}$ Porphyromonas was detected in $2 \%$ of the cases. We used RTF for collection and transfer of the specimens and Kanamycin Blood Agar for culture; where as in their study VMGA III was used for collection and transfer and Fastidious Anaerobic Agar was used for culture this may have been responsible for the difference in the percentage of organisms detected. In another study by Gomes et al ${ }^{10}$ where selective media was used for culture, Porphyromonas, was detected in $6.7 \%$ of the cases, Baumgartner et $\mathrm{al}^{11}$ reported a $9 \%$ detection of Porphyromonas gingivalis. In the recent studies conducted by Montagner et $\mathrm{al}^{12}$ and Tennert et $\mathrm{al}^{13}$ Porphyromonas gingivalis was detected in $5 \%$ and 9\% respectively in cases with primary endodontic infection. In the present study Prevotella intermedia was detected in $36.8 \%$ of symptomatic and in $9.5 \%$ of asymptomatic cases. Prevotella nigrescens was not detected in any of the samples. In a study by Montagner et $\mathrm{al}^{12}$ Prevotella was isolated in $25 \%$ of the cases from acute endodontic infections. In a studies by Gomes et al ${ }^{9,10}$ Prevotella was detected in $13 \%$ and $16.7 \%$ of the cases respectively, by culture. As reported by Baumgartner et al, ${ }^{14} \mathrm{PCR}$ is more accurate in differentiating between P. intermedia and P. nigrescens, which is difficult to be differentiated by culture. In our study Prevotella nigrescens was not detected. Prevotella and Porphyromonas organisms are associated with symptoms, however the same species can also be present with similar frequencies in asymptomatic cases. Hashioka et $\mathrm{al}^{15}$ in their study showed that Porphyromona were associated with symptoms in endodontic infections. Haapsalo et $\mathrm{al}^{16} \mathrm{in}$ their study showed that Prevotella was associated with symptomatic endodontic infections. However as is seen in the tables, the number of organisms, (CFU/ml), is more in the symptomatic group. The results of our study 
are in agreement with this. Enterococcus faecalis was not detected in any of the samples. This Gram-positive, facultative anaerobic cocco-bacteria is more frequently seen in failed endodontic therapy than in primary intraradicualar infections and even if present, it might be in the VBNC (Viable but not cultivable) state. ${ }^{17,18 .}$ In recent studies by Tennert et $\mathrm{al}^{13}$ and Xin et $\mathrm{al}^{19}, E$ faecalis was not identified in primary endodontic infections, however in the same study it was the most frequently isolated organism i.e, $33 \%$ in secondary endodontic infections. This explains the non detection of $E$ faecalis in our study, where samples were from primary endodontic infections,

Other organisms detected were Fusobacterium nucleatum, which is an anaerobic, Gram-negative, spindle shaped rod that is one of the most commonly encountered Gram-negative species in endodontic infections ${ }^{11}$ In our study it was detected in $42.1 \%$ of symptomatic and in 28.5 of asymptomatic cases. Gomez et $\mathrm{al}^{9}$ in the microbiological examination of infected root canals detected $F$. nucleatum in $11.7 \%$ of cases, by culture method. Vianna et $\mathrm{al}^{20}$ and Lana et al ${ }^{21}$ isolated $F$. nucleatum in $20 \%$ and $35 \%$ of the cases from teeth with necrotic pulps by culture techniques. It has also been associated with the development of severe forms of symptoms; according to a study conducted by Chavez de Paz et al. ${ }^{22}$ This finding is in accordance with the findings of our study. Actinobacillus actinomycetemcomitans (renamed as Aggregatibactor actinimycetemcomitans $)^{23}$, is a Gram-negative, facultatively anaerobic bacterium. It is an oral commensal and an opportunistic pathogen. It has a distinct racial bias and a range of potential virulence factors. ${ }^{24}$ It colonizes the human oral cavity and is a causative agent for aggressive periodontits. This organism is also detected in the samples from the root canal as seen in previous studies. ${ }^{25}$ In the present study A. actinomycetemcomitans was detected in $15.8 \%$ of symptomatic cases and $42.8 \%$ in asymptomatic cases. In a study by Rocas et al, ${ }^{25}$ this organism in detected in low frequencies where they used the Reverse-Capture Checkerboard Assay for identification of microbes in endodontic infections. In our study we have used the Dentaid media which improves the detection of A. actinomycetemcomitans. In the composition of Dentaid media, blood and serum have been omitted, hence reducing its cost and making it a more restrictive medium against the growth of other microorganisms with high nutritional requirements. ${ }^{26}$ This may be the reason for it being detected in more number of samples in our study. Klebsiella was detected in $4.7 \%$ (in only one of the asymptomatic cases) in our study. Klebsiella is non motile, gram negative, facultative anaerobic bacilli. In a previous study by Chaudhary et $\mathrm{al}^{27}$ it was detected as the most prevalent facultative anaerobic bacteria. Capocytophaga was detected in 1/21 $(4.7 \%)$ in asymptomatic cases. This is a Gram-negative, facultative anaerobe, fusiform, gliding bacilli and it forms part of the normal oral flora. They may occasionally cause systemic infections in the immuno-defecient cases. Sassone et al ${ }^{28}$ showed the detections of this organism in their study of primary endodontic infections. $\mathrm{Pal}$ et al, ${ }^{29}$ reported a case where Capnocytophaga was detected in a non-vital anterior tooth.

In $23.8 \%$ of samples from the asymptomatic group and $10.5 \%$ of samples from the symptomatic group no microbes were detected. Gomes et. al and Vianna et al have reported 'sterile' endodontic samples in culture based studies. ${ }^{9,1920}$ As the samples were taken from the main canal, bacteria from ramifications, apical deltas etc. cannot be detected. This can explain the 'sterile' culture in our study.

Table-1 Primary Endodontic Infections with Symptoms

\begin{tabular}{|c|c|c|c|c|c|c|}
\hline \multirow[t]{2}{*}{ Sample } & \multirow{2}{*}{$\begin{array}{l}\text { Enterococcus } \\
\text { faecalis } \\
\mathrm{CFU} / \mathrm{ml}\end{array}$} & \multirow{2}{*}{$\begin{array}{l}\text { Porphyromnas } \\
\text { gingivalis } \\
\text { CFU/ml }\end{array}$} & \multirow{2}{*}{$\begin{array}{l}\text { Prevotella } \\
\text { intermedia } \\
\text { CFU/ml }\end{array}$} & \multicolumn{3}{|c|}{ Other Organisms } \\
\hline & & & & $\begin{array}{l}\text { Streptococci } \\
\text { CFU/ml }\end{array}$ & $\begin{array}{l}\text { Fusobacterim } \\
\text { nucleatum } \\
\text { CFU/ml }\end{array}$ & $\begin{array}{l}\text { Aggregatibactor } \\
\text { actinomycetemcomitans } \\
\mathrm{CFU} / \mathrm{ml}\end{array}$ \\
\hline S-3 & _- & - & & $1,50,00,00$ & & 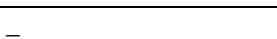 \\
\hline S-4 & - & $!, 00,000$ & 20,000 & $5,00,000$ & $3,00,000$ & - \\
\hline S-5 & - & - & - & $1,50,00,00$ & & - \\
\hline S-6 & _ & _ & _- & $8,00,000$ & $1,50.000$ & $1,00,000$ \\
\hline S-7 & - & - & 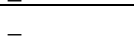 & $9,00,000$ & ( & - \\
\hline S-8 & - & $1,00,000$ & $2,00,000$ & $4,00,000$ & $1,00,000$ & - \\
\hline S-9 & - & _ & (1) & $6,00,000$ & - & - \\
\hline S-10 & _ & - & $1,00,000$ & $7,00,000$ & & _- \\
\hline S-11 & - & 4,000 & - & $7,00,000$ & $\overline{50,000}$ & - \\
\hline S-21 & - & 10,000 & 8,000 & 700,000 & - & - \\
\hline S-23 & - & & & 150,000 & 20,000 & 140,000 \\
\hline S-25 & - & - & - & - & - & - \\
\hline S-26 & - & - & - & - & - & - \\
\hline S-28 & - & 16,000 & 6,000 & 900,000 & - & - \\
\hline S-29 & - & - & 12,000 & 500,000 & 72,000 & - \\
\hline S-31 & - & 60,000 & 12,000 & 900,000 & - & - \\
\hline$S-32$ & - & & & $10,40,000$ & 16,000 & - \\
\hline S-35 & - & - & - & 900,000 & - & - \\
\hline S-39 & - & 16,000 & & 900,000 & 100,000 & 90,000 \\
\hline
\end{tabular}


Bacterial Identification in Symptomatic and Asymptomatic Endodontic Infections by Culture Method

\begin{tabular}{|c|c|c|c|c|c|c|}
\hline \multirow[t]{2}{*}{ Sample } & \multirow{2}{*}{$\begin{array}{l}\text { Enterococcus } \\
\text { faecalis } \\
\mathrm{CFU} / \mathrm{ml}\end{array}$} & \multirow{2}{*}{$\begin{array}{l}\text { Porphyromonas } \\
\text { gingivalis } \\
\text { CFU/ml }\end{array}$} & \multirow{2}{*}{$\begin{array}{l}\text { Prevotella } \\
\text { intermedia } \\
\mathrm{CFU} / \mathrm{ml}\end{array}$} & \multicolumn{3}{|c|}{ Other Organisms } \\
\hline & & & & $\begin{array}{l}\text { Streptococci } \\
\text { CFU/ml }\end{array}$ & $\begin{array}{l}\text { Fusobacterium } \\
\text { nucleatum } \\
\text { CFU/ml }\end{array}$ & $\begin{array}{l}\text { Aggregatibactor } \\
\text { actinomycetemcomitans } \\
\text { CFU/ml }\end{array}$ \\
\hline S-1 & $\ldots$ & . & 20,000 & $10,00,000$ & & 60,000 \\
\hline S-2 & - & $3,00,000$ & - & $6,00,000$ & $1,00,000$ & $2,40,000$ \\
\hline S-12 & - & 40,000 & _ & $9,00,000$ & - & - \\
\hline S-13 & _ & 8,000 & _- & $6,00,000$ & $1,50,000$ & 70,000 \\
\hline S-14 & $\ldots$ & 4,000 & _- & $9,00,000$ & 60,000 & 56,000 \\
\hline S-15 & $\ldots$ & $!, 50,000$ & _- & $9,00,000$ & $\ldots$ & Klebsiella-9,00,000 \\
\hline S-16 & _ & 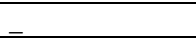 & _ & $4,00,000$ & - & $2,20,000$ \\
\hline S-17 & _- & - & _- & $8,00,000$ & _ & 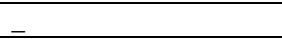 \\
\hline S-18 & _- & 36,000 & _- & $9,00,000$ & & $3,00,000$ \\
\hline S-19 & - & 8.000 & _ & $9,00,000$ & & $3,00,000$ \\
\hline $\mathrm{S}-20$ & $\ldots$ & 6,000 & - & $6,40,000$ & - & $1,30,000$ \\
\hline S-22 & - & 32,000 & 20,000 & 560,000 & 60,000 & - \\
\hline S-24 & - & - & - & 560,000 & 160,000 & 116,000 \\
\hline S-27 & - & - & - & 700,000 & - & - \\
\hline $\mathrm{S}-30$ & - & 8,000 & - & 360,000 & 120,000 & $\begin{array}{l}\text { Capnocytophaga } \\
60,000\end{array}$ \\
\hline S-33 & - & - & - & - & - & - \\
\hline S-34 & - & - & - & - & - & - \\
\hline S-36 & - & - & - & - & - & - \\
\hline S-38 & - & - & - & - & - & - \\
\hline $\mathrm{S}-40$ & - & - & - & - & - & $\begin{array}{l}\text { Pseudomonas } \\
\text { (contamination) }\end{array}$ \\
\hline S-42 & - & - & -- & - & - & - \\
\hline
\end{tabular}

Table-3, Percentage of various Organisms in Symptomatic and Asymptomatic Primary Endodontic Infection

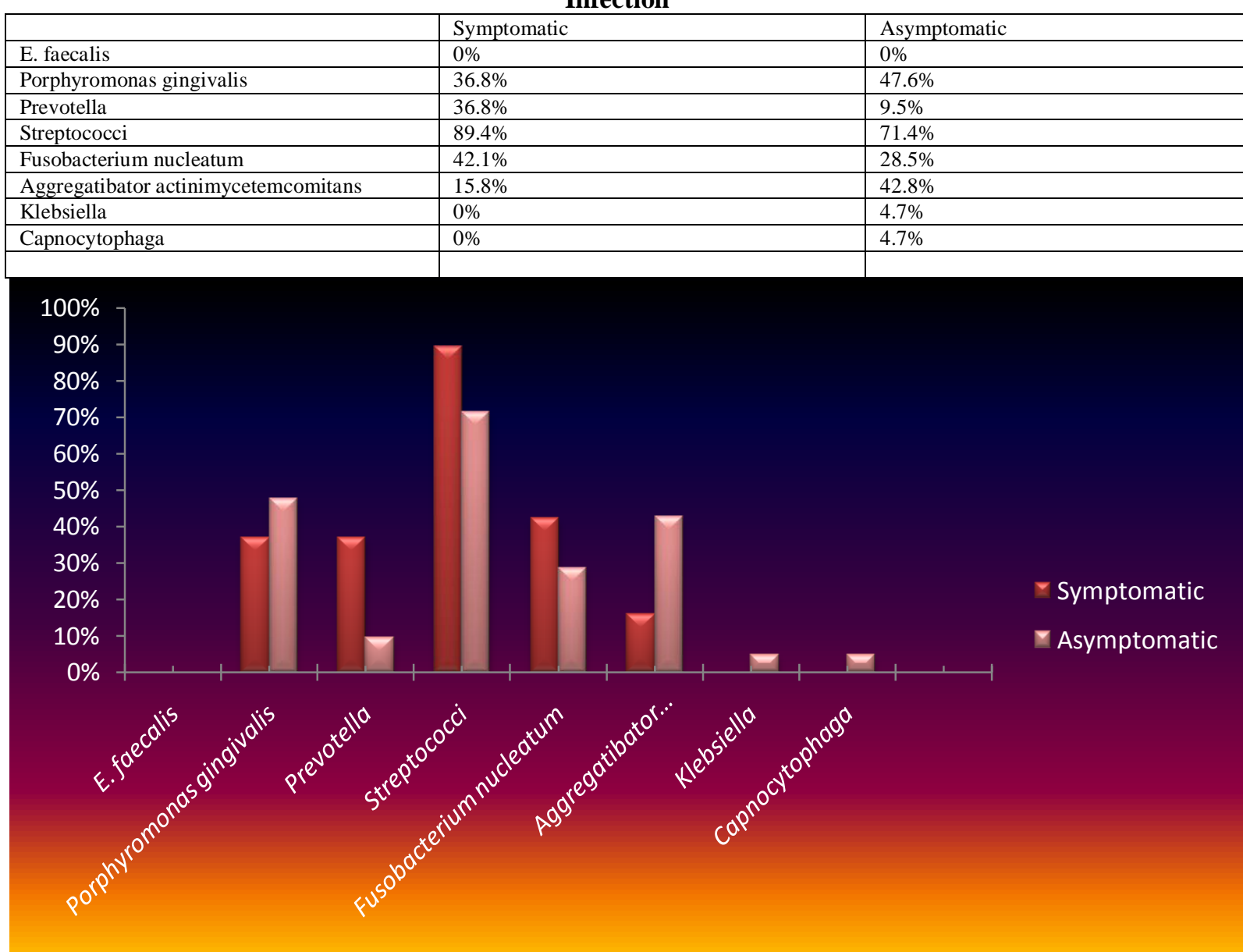

Figure-1, Graphic Representation of the Percentage of various Organisms in Symptomatic and Asymptomatic Primary Endodontic Infections 


\section{Conclusion}

The results of the present study confirm the polymicrobial nature of endodontic infections. Along with anaerobic Gram-negative bacteria, Gram-positive anaerobes and facultative anaerobes were also detected. Some of the targeted bacteria were not detected. The study shows no association between symptoms and certain bacterial species. There is no significant difference in the mean number of organisms detected between the two groups. Age and gender did not play any role in the mean number of organisms detected, between the two groups. The newer molecular techniques for the detection of microbes, will enable us to have a clear picture of this aspect. Future studies from multiple centers in India, with more number of samples and following the advanced molecular techniques will give a clear picture of the microbes in primary endodontic infections.

\section{References}

[1]. Sundqvist G. Association between microbial species in dental root canal infections. Oral Microbiol Immunol 1992, 7: 257-262.

[2]. Sathorn, P Parashos, HH Messer. How useful is root canal culturing in predicting treatment outcome? J Endod 2007;33:220-225.

[3]. Jose F Siqueira Jr. II Young Jung, I N Rocas, Chang Young Lee. Differences in prevalence of selected bacterial species in primary endodontic infections from two distinct geographic locations. Oral Surg Oral Med Pral Pathol Oral Radiol Endod 2005; 99:641 -647.

[4]. Schenkein H A, Burmeister J A, Koertge TE, Brooks CN, Best AM, Moore LV, et al. The influence of race and gender on periodontal microflora. J Periodontol. 1993,64: 292-296.

[5]. Sirinian G, Shimizu T, Sugar C, Slots J, Chen C. Periodontopathic bacteria in young healthy subjects of different ethnic backgrounds in Los Angeles. J Periodotol 2002;73:283-288.

[6]. Baumgartner JC, Siqueira JF Jr, Xia T, Rocas IN. Geographical differences in bacteria detected in endodontic infections using Polymerase chain reaction. J Endod 2004;30:141-144

[7]. Vartoukian SR, Palmer RM and Wade WG. Strategies for culture of 'unculturable' bacteria. 2010; FEMS Microbiol Lett 309:1-7.

[8]. Neringa Skucaite, Vytaute Peciuliene, Astra Vitkuskiene and Vita Machiuskiene. Susceptibility of endodontic pathogens to antibiotics in patients with symptomatic apical periodontitis, J Endod $201036.1611-1616$.

[9]. Gomes BPFA, Jacinto RC, Pinheiro ET, Sousa ELR, Zaia AA et al. Porphyromonas gingivalis, Porphyromonas endodontalis, Prevotella intermedia and Prevotella nigrescens in endodontic lesions detected by culture and by PCR. Oral Microbiol Immunol 2005: 20: 211-215.

[10]. Gomes BPFA, Pinheiro ET, Gade -Neto, Sousa ELR, Zaia Texeeira FJ et al. Microbial examination of infected dental root canals. Oral Microbiol Immunol 2004;19:71-76.

[11]. JC Baumgartner, B J Watkins, KS Bae, T Xia. Association of black-pigmented bacteria with endodontic infections .J Endod 1999,25;6:413- F Montagner, RC Jacinto, FGC Signoretti, VS Mattos, FS Grecca, BPFA Gomes

[12]. Beta-lactamic Resistance Profiles in Porphyromonas, Prevotella and Parvimonas Species Isolated from Acute Endodontic Infections. J Endod 2014;3:339-344.

[13]. C Tennert, M Fuhrmann, A Wittmer, L Karygianni, MJ Altenburger, E Hellwig, AA Ahmad. New Bacterial Composition in Primary and Persistent/Secondary Endodontic Infections with Respect to Clinical and Radiographic Finding. J Endod 2014;40:670-677.

[14]. Baumgartner J C, K. S Bae, T. Xia. Sodium dodecyl sulfate-polyacrylamine gel electrophoresis and PCR for differenciation of Prevotella intermedia and Prevotella nigrescens. J. Endod 1999, 25; 324-328.

[15]. Hashioka k, Yamasaki m, Nakane A, Horiba N and Nakamura M. The relationship between symptoms and anaerobic bacteria from root canals. 1992. J Endod,18; 558-561.

[16]. Haapsao M, Ranta H Ranta K and Shah H. Black pigmented Bacteroides spp. in human apical periodontitis. Infect Immune 1986 53:149-53.

[17]. M M, Tafi M C, Canepari P. Non-culturable Enterococcus faecalis cells are metabolically active and are capable of resuming active growth. Syst Appl Microbiol 1998, 21;333-339.

[18]. J.F.Siqueira Jr and I.N. Rocas. Diversity of Endodontic Microbiota Revisited. J Dent Res 2009, 88; 969-981

[19]. LI Xin, ZHU Xiao-fei, ZHANG Cheng-fei, Peter Cathro, CJ Seneviratne and SHEN Song. Endodontic bacteria from primary and persistent endodontic lesions in Chinese patients as identified by cloning and 16S ribosomal DNA gene sequencing. Chin Med J 2013;126 (4): 634-639

[20]. Vianna ME, Horz H-P, Gomes BPFA, Conrads G. Microarrays complement culture methods for identification of bacteria in endodontic infections. Oral Microbiol Immunol 2005: 20: 253-258

[21]. M. A. Lana, A. P. Ribeiro-Sobrinho, R. Stehling, G. D. Garcia, B. K. C. Silva, J. S. Hamdan, et al. Microorganisms isolated from root canals presenting necrotic pulp and their susceptibility in vitro. Oral Microbiol Immunol 2001: 16: 100-105

[22]. Chavez de Paz Villanueva LE. Fusobacterium nucleatum in endodontic flare-ups. Oral Surgery, Oral Medicine, Oral Pathology, Oral Radiology, and Endodontics. 2002;93: 179-83.

[23]. Neils Norskov-Lauritsen and Mogens Kilian. Reclassification of Actinobacillus actionomycetemcomitans, Haemophilus aphrophilus and Haemoplilus segnis to a new genus Aggregatibactor gen. Int J Syst Evol Microbiol 2006, 56; 2135-2146.

[24]. D Mythireyi and MG Krishnababa. Aggregatibactor actinomycetemcomitans an Aggressive Oral Bacteria - A Review. International J0urnal of Health Sciences and Research. 2012; 2: 107-115

[25]. I.N.Rocas J.F.Siqueira Jr et.al. Analysis of Symptomatic and Asymptomatic Primary Root Canal Infections in adult Norwegian Patients. J Endod 2011, 37: 1206-1212

[26]. Alsina M, Olle E, Frias J. Improved, low-cost selective culture medium for Actinobacillus actinomycetemcomitans. J Clin Microbiol. 2001,39;2: 509-13.

[27]. Chaudhry R. Kalra N. Talwar V. Thakur R. Anaerobic flora in endodontic infections. Indian J Med Res 1997, 105; $262-265$.

[28]. Sassone LM ${ }^{1}$, Fidel R, Faveri M, Fidel S, Figueiredo L, Feres M. Microbiological evaluation of primary endodontic infections in teeth with and without sinus tract. Int Endod J. 2008;41(6):508

[29]. D Pal, U K Chattopadhyay, G Das De and B S Dalal. Isolation of Capnocytophaga species from non-vital anterior teeth. Indian Journal of Pathology and Microbiology 1993, 36(1):28-31. 